\title{
Manipulation of Glassy State in Amorphous Selenium by Low-temperature Internal Friction Measurements
}

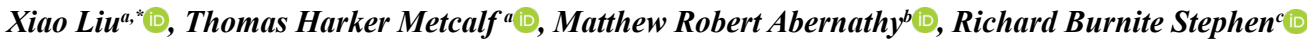 \\ ${ }^{a}$ Naval Research Laboratory, Washington, DC 20375, USA \\ ${ }^{b} N R C$ Research Associate, Washington, DC 20375, USA \\ 'University of Pennsylvania, Philadelphia, PA 19104, USA
}

Received: September 28, 2017; Revised: March 02, 2018; Accepted: April 20, 2018

\begin{abstract}
We have studied the thickness and quench-rate dependent internal friction of amorphous selenium ( $a$-Se) thin films deposited at room temperature. The internal friction of $a$-Se films exhibit a temperature independent plateau below $1 \mathrm{~K}$ followed by a broad maximum at $10 \mathrm{~K}$. The plateau, which is seen in almost all amorphous solids, is caused by dissipation by two-level tunneling systems (TLS), whose origin is still unknown. The maximum is caused by thermal relaxation over the same energy barrier that induces TLS. The internal friction and shear modulus are almost thickness independent from $100 \mathrm{~nm}$ to $10 \mu \mathrm{m}$. Unlike other elemental amorphous materials, the sufficiently low glass transition temperature $\left(\mathrm{T}_{\mathrm{g}}\right.$ ) of $a$-Se (only about $10 \mathrm{~K}$ above room temperature) allows in-situ quench-rate dependent study of TLS. The amorphous structure resets itself by a thermal equilibration cycle above $\mathrm{T}_{\mathrm{g}}$. We show that a faster quench rate freezes $a$-Se to a lower density structure with a higher TLS density and vice versa. The changes are reversible supporting a relationship between different quenched states and the density of TLS. Our study shows that $a$-Se can be a simple monatomic amorphous system to constrain models for the origin of TLS in amorphous solids.
\end{abstract}

Keywords: Internal friction, amorphous selenium, elastic modulus, speed of sound, tunneling systems, glass transition.

\section{Introduction}

One of the unsolved mysteries in condensed matter physics is the origin of the low-energy excitations in amorphous solids ${ }^{1,2}$. The existence of such ubiquitous lowenergy excitations enables amorphous solids to exhibit many universal properties that are absent in crystalline solids. While defect states in crystals usually have characteristic energies, the low-energy excitations of amorphous solids have a broad and almost energy independent distribution. They are the source of anomalous thermal, elastic and dielectric properties of amorphous solids ${ }^{2}$, such as: a linear temperature $(T)$ dependent specific heat, $T^{2}$ thermal conductivity below $1 \mathrm{~K}$, and an almost $T$ independent plateau in internal friction at a few $\mathrm{K}$. These properties are not only qualitatively but also quantitatively similar for almost all amorphous solids and some disordered crystalline solids, independent of their chemistry and details of structure ${ }^{3}$. The model of two-level tunneling systems (TLS) provides a phenomenological description for the low-energy excitations by assuming that a small number of atoms or groups of atoms with energetically similar configurations can tunnel between neighboring potential minima in an amorphous energy landscape ${ }^{4,5}$. However, the TLS model does not address the physical nature of the tunneling mechanisms. The challenge of identifying the microscopic origin of TLS stems from the universality of the excitations.
The interaction of TLS with elastic and electromagnetic waves causes energy dissipation in both mechanical and superconducting microwave resonators ${ }^{6,7}$. TLS are a source of noise and decoherence in superconducting circuits, such as superconducting quantum bits, photo detectors, quantum motion sensors, and SQUID multiplexers ${ }^{8}$. They are also a source of Brownian thermal noise of the optical thin films used to make the interferometer mirrors of gravitational wave detectors 9,10 . To understand the origin of TLS in order to eliminate them has become an urgent task. Recently, we found TLS can be almost completely removed in thin film amorphous silicon when it is deposited with a growth temperature of $400{ }^{\circ} \mathrm{C}{ }^{11,12}$. There are several theoretical explanations for the removal. It is possible that the tetrahedral bonding of silicon constrains the amorphous structure to make TLS less likely, as suggested by Phillips long ago ${ }^{5}$. It is however also possible that film deposition at a substrate temperature close to $85 \%$ of its respective $\mathrm{T}_{\mathrm{g}}$ makes the structure densely packed and close to an ultrastable state of glasses with higher mass density ${ }^{13}$. Recent work on the suppression of TLS in a deposited ultrastable state of glassy indomethacin may lend support to the ultrastable glass idea ${ }^{14}$.

Amorphous selenium $(a-\mathrm{Se})$ is a simple monatomic amorphous system that can be easily deposited as a thin film ${ }^{15}$. Its twofold covalence bonding enables it to form twisted chains of random lengths and monomer rings that are highly crystallization-resistant ${ }^{16}$. Its $\mathrm{T}_{\mathrm{g}}$ is conveniently $\sim 10 \mathrm{~K}$ above room temperature so that its structure can be

*e-mail: xiao.liu@nrl.navy.mil 
reset easily for quench-rate dependent study ${ }^{17}$. Because of its importance as a photo-sensitive semiconductor, many of its properties have been studied extensively ${ }^{18-20}$. Most importantly, a study of a twofold coordinated amorphous system will help us to distinguish models for the origin of TLS.

\section{Experimental and Results}

The $a$-Se thin films studied in this work were grown by thermal evaporation at room temperature. Film thicknesses were varied from $100 \mathrm{~nm}$ to $10 \mu \mathrm{m}$ in order to check any thickness dependent effect. Se beads (Goodfellow company, 99.999\%) were evaporated from an aluminum oxide crucible onto a double-paddle oscillator (DPO) made of single crystal silicon at a rate of $\sim 1 \mu \mathrm{m}$ per minute in a system with base pressure of $1 \times 10^{-6}$ Torr. A plastic chimney surrounded the evaporation boat extending to within $2 \mathrm{~cm}$ of the substrate holder; the substrate was rotated into position only after the source had come to temperature (just about Se melting temperature) and any residual gas had been swept out of the area by the Se vapor. The final thicknesses of the films are checked by spectroscopic ellipsometry after each deposition. A $112 \mathrm{~nm}$ thick film was used to study quench-rate dependence by repeatedly thermal cycling of the film to $313 \mathrm{~K}$. We observed crystallization of up to $20 \%$ of the total area after the third thermal cycle, which should have a minimal effect on the results to be presented. Some surface cracking was observed in the 2 and $10 \mu \mathrm{m}$ thick films, and more severe cracking in the $10 \mu \mathrm{m}$ thick film after repeated cooling; those thicker films were not used for the quench-rate study.

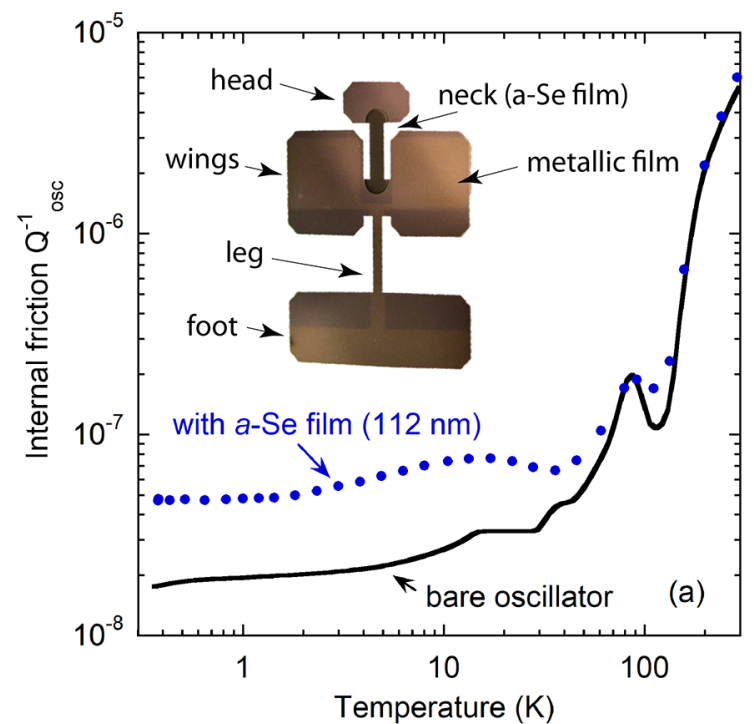

We measured the complex elastic properties, which include the shear modulus, $G_{\text {film }}$, relative change of speed of sound, $\Delta \boldsymbol{v} / \boldsymbol{v}_{0}$ and the internal friction, $Q^{-1}$, of $a$-Se films from below $1 \mathrm{~K}$ to room temperature by using the highest $Q$ resonance mode (the second anti-symmetric torsional mode, referred to as AS2) of the DPO ${ }^{21}$. The DPO consists of a head, a neck, two wings, a leg and a foot; see the insert of Fig. 1(a). The neck is the area where a thin film of $a$-Se to be studied is deposited. During resonance, the head and the wings vibrate out of phase, which leads to a torsional oscillation of the neck and the film while leaving the leg and the foot with little motion, minimizing the external loss. A metal film was deposited on the back of the DPO from the foot up to the wings (avoiding the neck and the head) for electrostatic drive and detection of its oscillation without comprising the high $Q$ of the mode; see the lightly colored area of the DPO in Fig. 1(a). Due to its excellent vibration isolation, the low background $Q^{-1}$ $\left(\sim 2 \times 10^{-8}\right)$ of AS2 ensures high sensitivity of the technique to extract elastic properties of thin films.

Addition of a film on the neck area leads to a measurable shift of a DPO's resonance frequency, from $f_{\text {sub }}$ to $f_{\text {osc }}$, and the internal friction, from $Q_{\text {sub }}^{-1}$ to $Q_{\text {sub }}^{-1}$. From the shift, the shear modulus, $G_{\text {film }}$, the internal friction, $Q_{f i l m}^{-1}$, and the relative change of speed of sound, $\left(\Delta \boldsymbol{v} / \boldsymbol{v}_{0}\right)_{\text {film }}=\left[\boldsymbol{v}_{\text {film }}-\boldsymbol{v}_{\text {film }}\left(T_{0}\right)\right] / \boldsymbol{v}_{\text {film }}$ of the film can be calculated through:

$$
\begin{gathered}
\frac{f_{\text {osc }}-f_{\text {sub }}}{f_{\text {sub }}}=\frac{3 G_{\text {film }} t_{f i l m}}{2 G_{\text {sub }} t_{\text {sub }}}, \\
Q_{\text {film }}^{-1}=\frac{G_{\text {sub }} t_{s u b}}{3 G_{\text {film }} t_{f i l m}}\left(Q_{\text {osc }}^{-1}-Q_{\text {sub }}^{-1}\right)+Q_{\text {osc }}^{-1},
\end{gathered}
$$

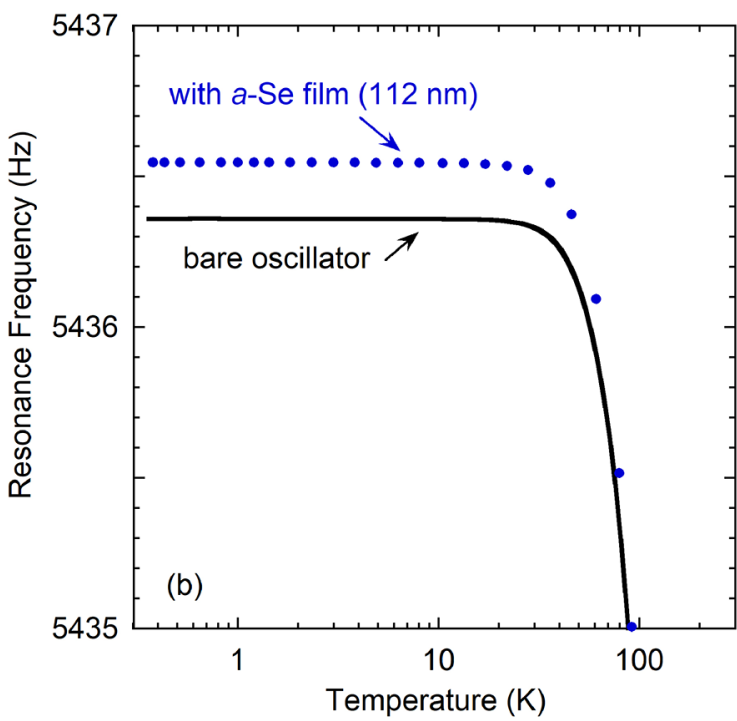

Figure 1. The internal friction, $Q_{o s c}^{-1}$, (a) and resonance frequency, $f$, (b) of a DPO before and after thermal evaporation of a $112 \mathrm{~nm}$ thick $a$-Se film. The inset in (a) shows the image of a DPO carrying an $a$-Se thin films in the neck area where most of the shear strain is concentrated. The light-colored area is coated with a metal film 
And

$$
\left(\frac{\Delta v}{v_{0}}\right)_{\text {film }}=\frac{G_{\text {sub }} t_{\text {sub }}}{3 G_{\text {film }} t_{f i m}}\left[\left(\frac{\Delta f}{f_{0}}\right)_{\text {osc }}-\left(\frac{\Delta f}{f_{0}}\right)_{\text {sub }}\right]+\left(\frac{\Delta v}{v_{0}}\right)_{s u b}
$$

where $t, G$, and $\Delta f / f_{0}=\left[f(T)-f\left(T_{0}\right)\right] / f\left(T_{0}\right)$ are thicknesses, shear moduli, and relative changes of resonance frequency, respectively of the oscillators, the substrates, and the films. $T_{0}$ is a reference temperature, taken as the lowest temperature point of the experiment. We also have $\mathrm{G}_{\text {sub }}=62 \mathrm{GPa}$, and $\mathrm{t}_{\text {sub }}=300 \mu \mathrm{m}$.

A representative temperature dependent internal friction and resonant frequency of a DPO before and after a deposition of a $112 \mathrm{~nm}$ thick $a$-Se film at room temperature is shown in Figs. 1(a) and (b), respectively. Both the internal friction increase and the frequency shift are proportional to $\mathrm{G}_{\text {film }} \mathrm{t}_{\text {film }}$. We calculate $G_{\text {film }}$ by subtracting the low temperature (below $30 \mathrm{~K}$ ) resonant frequency of the bare resonator from that with a film following Eq. (1); at low temperatures $f$ is relatively temperature independent. These low temperature values are typically about $5 \%$ higher than those measured at room temperature. To calculate the internal friction of the film, $Q_{f i m}^{-1}$, the background internal friction of the DPOs, $Q_{s u b}^{-1}$, was separately measured and subtracted from $Q_{\text {osc }}^{-1}$ following Eq. (2). For a bare DPO, thermoelastic loss dominates $Q_{\text {sub }}^{-1}$ at temperatures above $50 \mathrm{~K}$. The exact cause of loss at low temperature is not completely understood, but external losses are thought to dominate. The relative change of speed of sound can be determined from Eq. (3) by subtracting the relative change of resonance frequency of the bare DPO from that of the film laden DPO. The internal friction and the relative change of speed of sound data of three $a$-Se films are shown in Figs. 2(a) and (b), respectively. The low temperature $G_{\text {film }}$ data for the three films studied in this work are shown in the insert of Fig. 2(a). The measured $G_{\text {film }}$ values are in good agreement with those of bulk $a$-Se summarized in Ref. ${ }^{22}$. Although the values decrease slightly with increasing thickness, they are still within the error bar of the technique.

The relaxation scattering of elastic waves by TLS contributes to the temperature independent plateau in internal friction, $Q_{0}^{-1}$, which is described by the tunneling strength $C$ in the TLS model, as $^{3}$

$$
Q_{0}^{-1}=\frac{\pi}{2} C,
$$

where $C$ is defined as

$$
C=\frac{\bar{P} \gamma^{2}}{\rho v^{2}}
$$

and $\bar{P}$ is the spectral density of the tunneling states, $\gamma$ the energy with which they are coupled to phonons, $\rho$ the mass density, and $v$ the speed of sound. $Q_{0}^{-1}$ varies roughly within a range of about one order of magnitude for most glasses and amorphous solids. For comparison with a prototypical amorphous solid, Fig. 2(a) shows the $Q_{\text {fim }}^{-1}$ of a $107 \mathrm{~nm}$ thick dry thermal oxide $\left(\mathrm{a}-\mathrm{SiO}_{2}\right)$ film grown directly on a DPO at $1100^{\circ} \mathrm{C}$. The universal magnitude of $Q_{0}^{-1}$ is surprising given the individual parameters that constitute $C$, like mass density $\rho$ or elastic constants $\rho v^{2}$, may vary by many orders of magnitude ${ }^{3}$. Thus, a measurement of $Q_{0}^{-1}$ directly reveals the universality of the low-energy excitations as well as the spectral density of TLS.

Fig. 2(a) shows that $a$-Se films, with thicknesses differing by two orders of magnitude, have approximately the same $Q_{0}^{-1}$, similar to that of $a-\mathrm{SiO}_{2}$. The broad maximum following the plateau is caused by thermal activation over the same barrier that induces the TLS. The difference between $a$-Se and $a-\mathrm{SiO}_{2}$ is that the maximum locates at lower temperature for $a$-Se, presumably due to its softer structure. Again, any thickness dependence in $Q_{\text {fim }}^{-1}$ is insignificant, indicating bulk properties still prevail for a film as thin as $100 \mathrm{~nm}$.
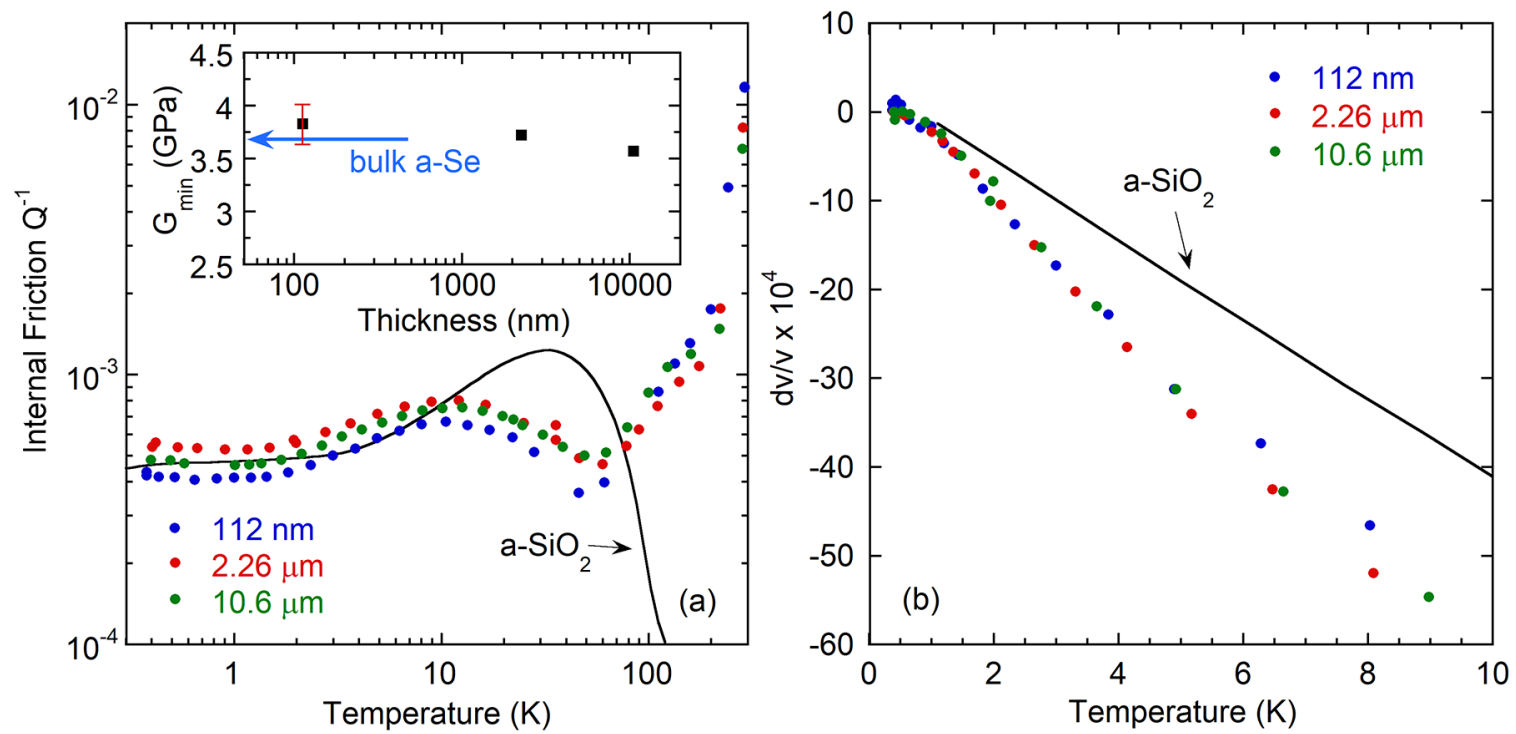

Figure 2. The internal friction $Q_{\text {film }}^{-1}$ (a) and the relative change of speed of sound $\Delta v / v_{0}$ (b) of three $a$-Se films with different thicknesses. The data of a $107 \mathrm{~nm}$ thick dry thermal oxide (a-SiO$)$ film are shown in both (a) and (b) for comparison. The insert shows their shear modulus $\mathrm{G}_{\text {film }}$ vs. thickness. 
TLS can also be indirectly accessed by the real part of the elastic constant, which yields the variation of the speed of sound with temperature. The characteristic features of $\Delta v / v_{0}$ by resonant and relaxational scattering by TLS occur at lower temperatures than we can access in this work. Fortunately, $\Delta v / v_{0}$ is known to vary linearly with temperature in amorphous solids from a few $\mathrm{K}$ up to a few tens of $\mathrm{K}^{23}: \Delta v / v_{0}=-\beta\left(T-T_{0}\right)$, where $\beta$ is also proportional to $C$ and $Q_{0}^{-1}$. This dependence at higher $\mathrm{T}$ is understood as the thermally activated relaxation rate of the TLS dominating the quantum tunneling rate ${ }^{24}$. The $a-\mathrm{SiO}_{2}$ data of $\Delta v / v_{0}$ are also shown in Fig. 2(b) for comparison. The slightly different slopes of the two materials are not unusual for amorphous solids.

The quench-rate dependent $Q_{\text {film }}^{-1}$ is shown in Fig. 3(a) and (b). The response of both internal friction plateau and the broad maximum following it to different quench rate is obvious, although the total effect is about $10 \%$. The as-deposited film is in a state that is equivalent to a quench rate between 0.017 and $1.9 \mathrm{~K} / \mathrm{min}$. An anneal at $313 \mathrm{~K}$, which is above $\mathrm{T}_{\mathrm{g}}$ of $a$-Se, resets the amorphous structure to an equilibrium state. This makes the quench-rate study completely reversible as demonstrated in Fig. 3(b). Different quench rates freeze the structure into a different quasi-equilibrium state corresponding to a different fictive temperature. This is an unambiguous demonstration that TLS are generated in the process of glass formation when an undercooled liquid drops out of thermal equilibrium ${ }^{25}$. The same process produces most of the glasses seen in nature. As a change in quench rate more than two orders of magnitude only induces about $10 \%$ change in $Q_{0}^{-1}$ or $C$, which is proportional to TLS density (see Eqs. 4 and 5), our result also demonstrates the insensitivity of the TLS in response to a structure change. This may be further related to the unknown mechanisms which make the low energy excitations in amorphous solids universal.

\section{Discussion}

There has been a great interest in removing TLS in amorphous thin films in a variety of applications. Our work is a demonstration that annealing and slow quenching helps, but the effect is limited, probably due to the universality of the states. It is possible that no matter how slow the cooling is during quenching, the structural relaxation rate will eventually become slower than the cooling rate to drive the system out of thermal equilibrium with frozen-in disorder. Slow quenching may not be a feasible avenue to remove TLS. A study shows that TLS density hardly changes even if a glass is aged longer than 100 million years ${ }^{26}$.

However, two recent efforts to remove TLS in amorphous systems have been encouraging. One is ultrastable glasses of indomethacin deposited by thermal evaporation at $85 \%$ of its $\mathrm{T}_{\mathrm{g}}{ }^{14}$. The authors find that the stable indomethacin films do not possess the linear- $T$ dependent specific heat at low
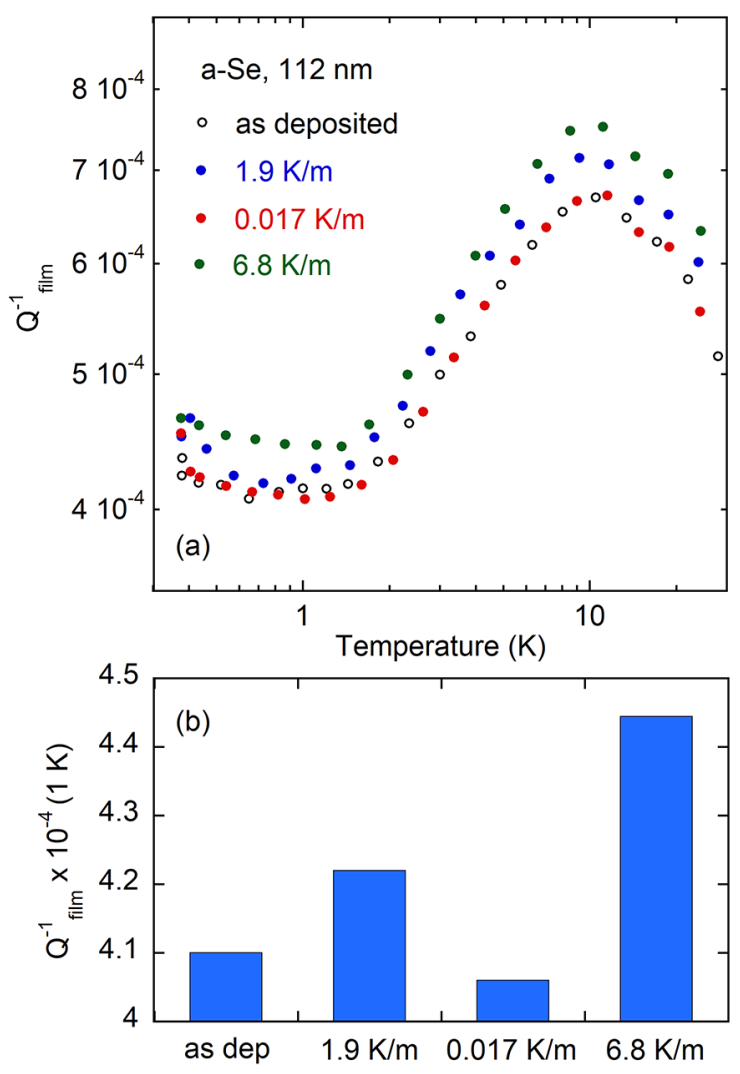

Figure 3. The internal friction $Q_{\text {film }}^{-1}$ of the $112 \mathrm{~nm}$ thick $a$-Se before and after a series of quench starting from $313 \mathrm{~K}$ : (a) the temperature dependence; and (b) compasrison of internal friction plateau values at $1 \mathrm{~K}$

temperatures, which is characteristic of TLS. Stable glasses take advantage of the higher mobility of surface molecules ${ }^{27}$, and utilize slow deposition rates and substrate temperatures slightly below $\mathrm{T}_{\mathrm{g}}(\sim 85 \%)$, so that a freshly deposited layer can optimize its local structure before being constrained by later layers, bypassing the long bulk thermal relaxation processes. This method allows creation of films that are considerably denser and remarkably more stable than ordinary glasses, reminiscent of ideal glasses. The other is amorphous silicon films deposited by $e$-beam evaporation at a substrate temperature of $673 \mathrm{~K}^{11,12}$ as mentioned earlier in the introduction. In this case, a disappearance of both the linear- $T$ dependent specific heat and internal friction plateau at low temperatures has been observed. Although the exact cause for the disappearance of TLS is not known, this fourfold covalently bonded monatomic amorphous thin film system is deposited at a temperature also close to its $\mathrm{T}_{\mathrm{g}}(\sim 70 \%)^{28}$. As we show that TLS are linked to the frozen-in states in $a$-Se, a study of substrate temperature dependence of TLS density would help us to answer the following questions: if vapor deposited $a$-Se can become ultrastable, and if TLS can be removed from this twofold covalently bonded amorphous system. This would help to examine whether an ultrastable state of a glass is sufficient to remove TLS, or other structural features like tetrahedral bonding are necessary to suppress TLS in amorphous solids. 


\section{Conclusions}

In this work we show that the internal friction and the relative change of speed of sound of $a$-Se films exhibit the typical behavior of amorphous solids. The elastic properties are mostly bulk-like as they depend only weakly on film thicknesses from $100 \mathrm{~nm}$ to $10 \mu \mathrm{m}$. Our results demonstrate that TLS respond to quench rate reversibly as the film is cooled from above its $\mathrm{T}_{\mathrm{g}}$, indicating that TLS are associated with glass formation when an undercooled liquid drops out of thermal equilibrium. It is thus possible to further reduce TLS density by making glasses that approach their thermal equilibrium. However, the relative small change of about $10 \%$ in TLS density for over two orders of magnitude change in quench rate demonstrates the limit of slow quenching to modify TLS. Recent work on reaching ultrastable states of glasses by depositing thin film amorphous materials at a substrate temperature close to $85 \%$ of its respective glass transition temperature may have provided a feasible avenue to reach equilibrated stable states of glasses which may be free of the universal low energy excitations.

\section{Acknowledgments}

This work was supported by the Office of Naval Research and by the National Science Foundation through the University of Pennsylvania Materials Research Science and Engineering Center, DMR-1120901.

\section{References}

1. Zeller RC, Pohl RO. Thermal Conductivity and Specific Heat of Noncrystalline Solids. Physical Review B. 1971;4(6):2029-2041.

2. Phillips WA. Amorphous Solids: Low Temperature Properties. Berlin: Springer Verlag; 1981.

3. Pohl RO, Liu X, Thompson E. Low-temperature thermal conductivity and acoustic attenuation in amorphous solids. Review of Modern Physics. 2002;74(4):991-1013.

4. Anderson PW, Halperin BI, Varma CM. Anomalous lowtemperature thermal properties of glasses and spin glasses. Philosophical Magazine. 1972;25(1):1-9.

5. Phillips WA. Tunneling states in amorphous solids. Journal of Low Temperature Physics. 1972;7(3-4):351-360.

6. Park YS, Wang HL. Resolved-sideband and cryogenic cooling of an optomechanical resonator. Nature Physics. 2009;5(7):489493.

7. Martinis JM, Cooper KB, McDermott R, Steffen M, Ansmann M, Osborn KD, et al. Decoherence in Josephson Qubits from Dielectric Loss. Physical Review Letters. 2005;95(21):210503.

8. Eckstein JN, Levy J. Materials issues for quantum computation. MRS Bulletin. 2013;38(10):783-789.

9. Harry GM, Gretarsson AM, Saulson PR, Kittelberger SE, Penn SD, Startin WJ, et al. Thermal noise in interferometric gravitational wave detectors due to dielectric optical coatings. Classical and Quantum Gravity. 2002;19(5):897-917.
10. Mitrofanov VP, Chao S, Pan HW, Kuo LC, Cole G, Degallaix $\mathrm{J}$, et al. Technology for the next gravitational wave detectors. Science China Physics, Mechanics \& Astronomy. 2015;58:120404.

11. Queen DR, Liu X, Karel JE, Metcalf TH, Hellman F. Excess specific heat in evaporated amorphous silicon. Physical Review Letters. 2013;110(13):135901.

12. Liu X, Queen DR, Metcalf TH, Karel JE, Hellman F. Hydrogenfree amorphous silicon with no tunneling states. Physical Review Letters. 2014;113(2):025503.

13. Queen DR, Liu X, Karel JE, Jacks HC, Metcalf TH, Hellman F. Two-level systems in evaporated amorphous silicon. Journal of Non-Crystalline Solids. 2015;426:19-24.

14. Pérez-Castañeda T, Rodríguez-Tinoco C, Rodríguez-Viejo J, Ramos MA. Suppression of tunneling two-level systems in ultrastable glasses of indomethacin. Proceedings of the National Academy of Sciences. 2014;111(31):11275-11280.

15. Grimley RT, Grindstaff QG, DeMercurio TA, Forsman JA. A study of the selenium vapor system by angular distribution mass spectroscopy. Journal of Physical Chemistry. 1982;86(6):976-982.

16. Goldan AH, Li C, Pennycook SJ, Schneider J, Blom A, Zhao W. Molecular structure of vapor-deposited amorphous selenium. Journal of Applied Physics. 2016;120(13):135101.

17. Larmagnac JP, Grenet J, Michon P. Glass transition temperature dependence on heating rate and on ageing for amorphous selenium films. Journal of Non-Crystalline Solids. 1981;45(2):157-168.

18. Stephens R. The viscosity and structural relaxation rate of evaporated amorphous selenium. Journal of Applied Physics. 1978;49(12):5855-5864.

19. Málek J, Svoboda R, Pustková P, Cicmanec P. Volume and enthalpy relaxation of a-Se in the glass transition region. Journal of Non-Crystalline Solids. 2009;355(4-5):264-272.

20. Gulbiten O, Mauro JC, Lucas P. Relaxation of enthalpy fluctuations during sub- $\mathrm{T}_{\mathrm{g}}$ annealing of glassy selenium. Journal of Chemical Physics. 2013;138(24):244504.

21. Liu X, Morse SF, Vignola JF, Photiadis DM, Sarkissian A, Marcus $\mathrm{MH}$, et al. On the modes and loss mechanisms of a high Q mechanical oscillator. Applied Physics Letters. 2001;78(10):1346-1348.

22. Etienne S, Guenin G, Perez J. Ultrasonic studies of the elastic coefficients of vitreous selenium about $\mathrm{T}$. Journal of Physics D: Applied Physics. 1979;12(12):2189-2202.

23. Bellessa G. Frequency and Temperature Dependence of the Sound Velocity in Amorphous Materials at Low Temperatures. Physical Review Letters. 1978;40(22):1456-1459.

24. Tielbürger D, Merz R, Ehrenfels R, Hunklinger S. Thermally activated relaxation processes in vitreous silica: An investigation by Brillouin scattering at high pressures. Physical Review $B$. 1992;45(6):2750-2760.

25. Löhneysen Hv, Rüsing H, Sander W. Structural Relaxation Effects on the Low-Temperature Properties of Vitreous Silica. Zeitschrift für Physik B Condensed Matter. 1985;60(2-4):323-330.

26. Pérez-Castañeda T, Riobóo RJ, Ramos. Two-level systems and boson peak remain stable in 110-million-year-old amber glass. Physical Review Letters. 2014;112(16):165901. 
27. Zhu L, Brian CW, Swallen SF, Straus PT, Ediger MD, Yu L. Surface Self-diffusion of an organic glass. Physical Review Letters. 2011;106(25):256103.
28. Hedle A, Klaumünzer SL, Wesch W. Amorphous silicon exhibits a glass transition. Nature Materials. 2004;3(11):804809 . 